\title{
Processing Graded Feedback: Electrophysiological Correlates of Learning from Small and Large Errors
}

\author{
Caroline Di Bernardi Luft ${ }^{1,2}$, Emilio Takase ${ }^{2}$, \\ and Joydeep Bhattacharya ${ }^{1}$
}

\begin{abstract}
Feedback processing is important for learning and therefore may affect the consolidation of skills. Considerable research demonstrates electrophysiological differences between correct and incorrect feedback, but how we learn from small versus large errors is usually overlooked. This study investigated electrophysiological differences when processing small or large error feedback during a time estimation task. Data from highlearners and low-learners were analyzed separately. In both high- and low-learners, large error feedback was associated with higher feedback-related negativity (FRN) and small error feedback was associated with a larger P300 and increased amplitude over the motor related areas of the left hemisphere. In addition, small error feedback induced larger desynchronization in the alpha and beta bands with distinctly different
\end{abstract}

\section{INTRODUCTION}

Learning motor skills is often considered implicit: The learning is slow and incremental, and the learned knowledge is difficult to express verbally. Furthermore, a lot of practice is needed to learn a skill (Diedrichsen, White, Newman, \& Lally, 2010), and feedback from various sources is often required for learning to take place (Masters, Maxwell, \& Eves, 2009). Here, feedback can be conceptualized as an outcome of an action that can be obtained from various sources, from direct sensory registrations, like looking where the ball goes after kicking it, to more intricate ones, like the coach's verbal feedback about a specific movement. As learning crucially depends on the type of information used to guide performance (Wolpert, Diedrichsen, \& Flanagan, 2011), feedback is widely investigated in the research on learning (Wulf, Chiviacowsky, Schiller, \& Avila, 2010; Wu \& Zhou, 2009; Kawashima et al., 2000; Burkhard, Patterson, \& Rapue, 1967).

An often-studied measure of how feedback is processed in the brain is the feedback-related negativity (FRN), a negative deflection in the ERP that starts $\sim 150 \mathrm{msec}$ and peaks between 230 and $330 \mathrm{msec}$ following an incorrect or error feedback (Holroyd \& Krigolson, 2007;

\footnotetext{
${ }^{1}$ University of London, ${ }^{2}$ University of Santa Catarina

topographies between the two learning groups: The highlearners showed a more localized decrease in beta power over the left frontocentral areas, and the low-learners showed a widespread reduction in the alpha power following small error feedback. Furthermore, only the high-learners showed an increase in phase synchronization between the midfrontal and left central areas. Importantly, this synchronization was correlated to how well the participants consolidated the estimation of the time interval. Thus, although large errors were associated with higher FRN, small errors were associated with larger oscillatory responses, which was more evident in the high-learners. Altogether, our results suggest an important role of the motor areas in the processing of error feedback for skill consolidation.

Luu, Tucker, Derryberry, Reed, \& Poulsen, 2003; Miltner, Braun, \& Coles, 1997) or punishments like monetary losses (Cohen \& Ranganath, 2007; Hajcak, Moser, Holroyd, \& Simons, 2007). The FRN has a midfrontal topography and is likely to be generated in the ACC, especially the dorsal portion (Balconi \& Scioli, 2011; Santesso et al., 2012; Potts, Martin, Kamp, \& Donchin, 2011; Zhou, Yu, \& Zhou, 2010; Ladouceur, Dahl, \& Carter, 2007; Luu et al., 2003; Van Veen \& Carter, 2002; Carter et al., 1998; Miltner et al., 1997).

Most studies that have investigated feedback processing by FRN have provided feedback in a categorical format, for example, correct versus incorrect, yet in reallife learning situations, feedback information is mostly obtained in a graded or exact form. Nonetheless, some studies with gambling tasks comparing different magnitudes of reward have found that the FRN reflects an evaluation of whether an event was good or bad rather than how good or bad they were (Hajcak, Moser, Holroyd, \& Simons, 2006; Yeung \& Sanfey, 2004; Gehring \& Willoughby, 2002). There are only few studies analyzing the ERPs in response to graded feedback. They suggest that the FRN is not sensitive to the magnitude of the performance feedback (Mars, Bruijn, Hulstijn, Miltner, \& Coles, 2004; Tucker, Luu, Frishkoff, Quiring, \& Poulsen, 2003), and the results remain inconclusive (Walsh \& Anderson, 2012), with some studies indicating that the 
FRN is sensitive to the magnitude of the reward (Kreussel et al., 2012, 2013; Bellebaum, Polezzi, \& Daum, 2010; Wu \& Zhou, 2009), and others not (Hajcak et al., 2006; Sato et al., 2005; Yeung \& Sanfey, 2004). Furthermore, these studies provided graded feedback belonging to a limited number of categories (i.e., fast, medium, slow), yet in many tasks (i.e., sensorimotor), the feedback is available in a more finely graded format. For example, when throwing a dart, visual feedback indicates how far it has landed from the target, not just the categorical result (hit or miss, or good, medium and bad hit or miss). Despite the importance of finely graded feedback in many learning situations, the neural representation of finely graded feedback remains largely uncharacterized.

As stated earlier, the FRN is an ERP component that is obtained after signal averaging across trials. The FRN, therefore, cannot capture time-varying neural oscillations and intersite synchronizations, which are not locked to the onset of feedback. Recent evidence indeed suggests that the oscillatory correlates of feedback processing can shed new light on the understanding of how the feedback is processed for learning (Luft, Nolte, \& Bhattacharya, 2013; Cavanagh, Zambrano-Vazquez, \& Allen, 2012; HajiHosseini, Rodriguez-Fornells, \& Marco-Pallares, 2012; Cohen, Wilmes, \& Vijver, 2011; van de Vijver, Ridderinkhof, \& Cohen, 2011; Marco-Pallares et al., 2008; Cohen, Elger, \& Ranganath, 2007). For instance, negative feedback is associated with higher midfrontal theta $(4-8 \mathrm{~Hz})$ power, whereas positive feedback is associated with an increase in the beta (around 15-30 Hz) power (HajiHosseini et al., 2012; van de Vijver et al., 2011; Marco-Pallares et al., 2008; Cohen et al., 2007). Furthermore, synchronization between midfrontal (midline electrodes FCz, Fz, Fpz) and prefrontal regions (electrodes $\mathrm{F} 5 / \mathrm{F} 6$ ) is increased during error identification and subsequent adjustments (van de Vijver et al., 2011; Cavanagh, Frank, Klein, \& Allen, 2010; Cavanagh, Cohen, \& Allen, 2009). However, to our knowledge, there is currently no study characterizing the oscillatory correlates of feedback processing in response to different magnitudes of graded feedback.

In a recent study (Luft et al., 2013), we observed that high-learners, that is, participants who quickly and reliably learned a time interval estimation task, demonstrated larger phase synchronization between left central (sensorimotor) and midfrontal and between midfrontal and prefrontal areas (electrodes F5/F6) in the theta frequency range following categorical feedback. In addition, we found that high-learners demonstrated larger midfrontal theta oscillations in response to incorrect feedback compared with low-learners. In this study, we adopted the same experimental paradigm, but with a modified feedback format, where feedback was provided in a finely graded manner. Specifically, instead of receiving categorical outcome feedback (i.e., correct or incorrect), the participants were informed exactly how many milliseconds their time estimations deviated from the target.
This allowed us to compare the brain responses (ERPs, oscillations, and intersite synchronization) to feedback with identical valence but specific gradation. Therefore, this study aimed to address the following questions: (1) What are the differences in the electrophysiological correlates of processing small and large error feedback? (2) Is there a difference between high- and low-learners in relation to the way they process graded feedback?

Following earlier studies investigating the FRN in response to various feedback magnitudes (Kreussel et al., 2012, 2013; Bellebaum et al., 2010; Wu \& Zhou, 2009; Hajcak et al., 2006; Sato et al., 2005; Yeung \& Sanfey, 2004), our main prediction was that small error feedback would be processed as "correct," whereas large errors would have the same neural correlates of "incorrect." Our second prediction was that the high-learners would differ from low-learners in relation to their neural responses to small and large error feedback. Finally, following our previous study (Luft et al., 2013), we also predicted that the high-learners will show higher intersite connectivity in response to feedback compared with the low-learners.

\section{METHODS}

\section{Participants}

Thirty-two healthy adults (mean age $=24.4$ years, $S D=5.08$ years, 11 men, 2 left-handed) with normal hearing (self-reported) and normal or corrected-tonormal vision participated in the experiment. The sample was equally divided into two groups according to the performance on the experimental task, as explained later. This procedure resulted in two groups: the high-learners $(n=16$, mean age $=24.8$ years, 5 men $)$ and the lowlearners $(n=16$, mean age $=24$ years, 6 men $)$. The participation was entirely voluntary, and no one received any payment or incentive for taking part in this study. All participants provided written informed consent. The study was approved by the local ethics committee of the Department of Psychology at Goldsmiths, University of London, and conducted in accordance with the Declaration of Helsinki.

\section{Experimental Task}

We used a modified version of a time estimation task (Miltner et al., 1997), in which a participant is required to estimate the time interval of $1 \mathrm{sec}$ after the presentation of a sound by pressing a button. In our study, the participants were required to estimate the time interval of $1.7 \mathrm{sec}$. The time interval was modified from the original $1 \mathrm{sec}$ (Miltner et al., 1997) to $1.7 \mathrm{sec}$ for the two following reasons: (1) With integer second durations, participants tend to rely more on counting than on feedback while trying to adjust their estimation; (2) The brain mechanisms 
for sub- and suprasecond time estimations are different, with the suprasecond durations less automatic than the subseconds ones (Lewis \& Miall, 2003). The experimental paradigm was similar to our previous study (Luft et al., 2013) apart from the fact that the feedback was provided as graded information which indicated the exact difference (in msec) between the participant's estimation and the target (1700 msec).

Each trial started with a fixation cross presented for a time interval varying randomly between 300 and $900 \mathrm{msec}$ and was followed by an auditory beep $(800 \mathrm{~Hz}, 50 \mathrm{msec})$, which indicated the beginning of the time estimation. Participants were required to press a button with their right thumb (including the left-handed participants) whenever they thought $1.7 \mathrm{sec}$ had elapsed. Six hundred milliseconds after the button press, participants received a feedback indicating the magnitude (the exact difference [in $\mathrm{msec}$ ] between the participant's estimation and the target) and the sign (a negative sign indicated that the estimation was shorter, whereas a positive sign indicated longer than the target) of their estimation error. The feedback was presented for $1 \mathrm{sec}$.

To investigate the learning, we included six blocks of 20 trials each without any valid feedback where the word "OK" was presented for $1 \mathrm{sec}$. There were six feedback blocks, with 80 trials each, interspersed with six nonfeedback blocks with 20 trials each. In total, there were 480 feedback trials and 120 nonfeedback trials. The experiment started with a nonfeedback block and ended with a nonfeedback block. The participants had small breaks between each block and a longer break in the middle of the task. Because of this longer break in the middle of the task, the second half of the experiment started with a feedback block (making two feedback blocks in succession). The task structure (feedback and nonfeedback blocks) is presented along with the performance in Figure 1 (areas shown in gray represent the nonfeedback blocks).

The feedback trials were divided into two types, large and small error, based on the percentiles of the absolute error magnitude for each block. We chose the bottom 25 percentile as the small error and the top 25 percentile as the large error feedback condition within each block for each participant. This process ensured an identical number of large and small error trials in each block for each participant and allowed us to analyze the differences related to the use of feedback as information for performance adjustment without confounding with expectation associated with lower errors in the final blocks. The participants were divided into two groups based on their performance in the last three nonfeedback blocks. The main motivation to use the last three nonfeedback blocks was to derive a measure that was independent of analyzed brain responses and could reflect the consolidation of the trained time interval. The last three blocks were in the second half of the task, when the performance was supposed to be stable (Figure 1).

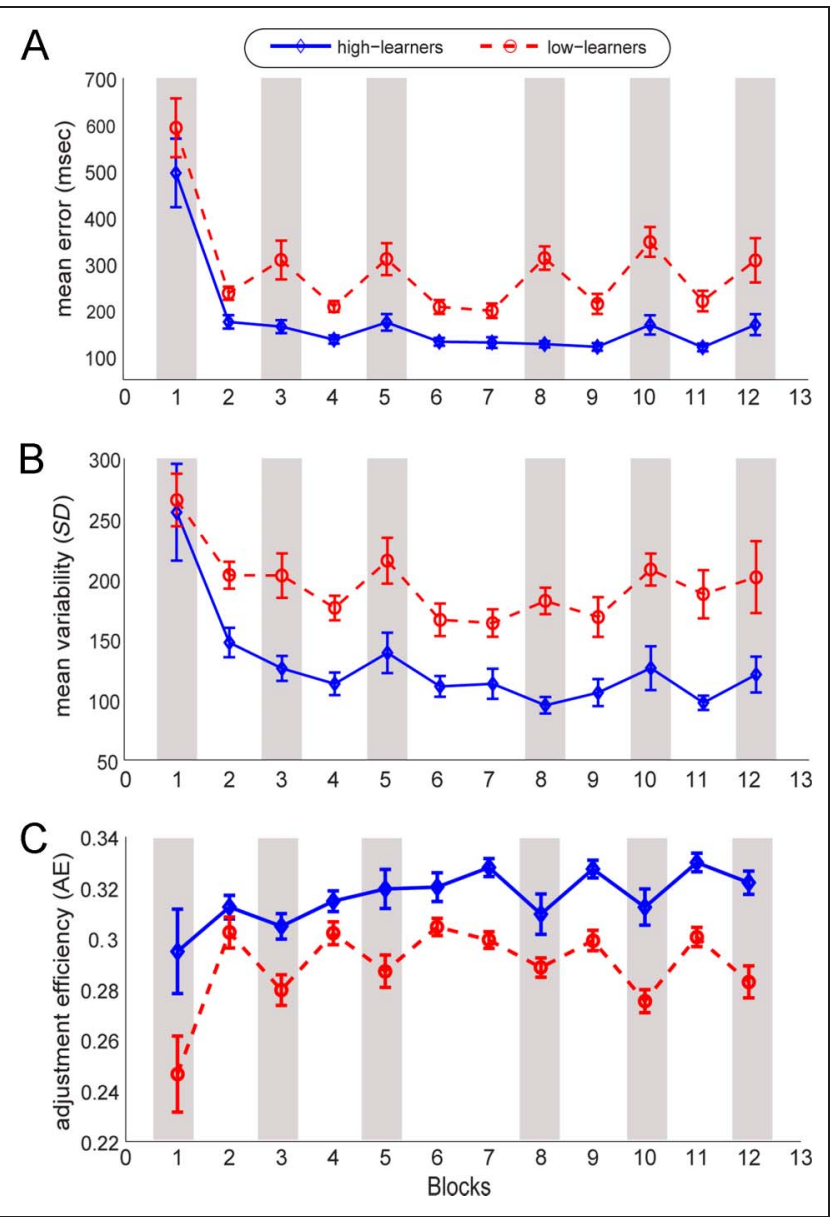

Figure 1. Performance in each block of the task. Performance measures of the two groups, high-learners (blue) and low-learners (red), across two types of blocks, feedback and nonfeedback (gray). Performance was evaluated by (A) absolute error magnitude, (B) response variability, and $(\mathrm{C}) \mathrm{AE}$.

Moreover, the fact that the participants did not receive any feedback ensured that their performances during these blocks did reflect the true learning of the time estimation, but not an adjustment to the feedback. We calculated two measures for the evaluation of learning: the error magnitude (the average absolute error of the last three nonfeedback blocks) and the response variability (the average of standard deviation of absolute errors within each block). These two measures were first normalized between 0 to 1 and then averaged. A median split on this combined measure was used to divide the participants into two groups. The participants in the top $50 \%$ of this combined measure were classified as the low-learners group (with larger error and high response variability), and the participants in the bottom $50 \%$ as the highlearners group (with smaller error and low response variability). The performance variability was used, in addition to the absolute error, as a criterion for splitting the groups because consistency in performance is a major hallmark of learning (Wolpert et al., 2011; Reis et al., 2009). 
We also analyzed the efficiency of performance adjustment based on the feedback by introducing a measure, called the adjustment efficiency (AE), calculated as

$$
\mathrm{AE}=\frac{e(i-1)-e(i)}{e(i-1)}=\frac{\Delta e}{e(i-1)}
$$

where $e(i)$ is the error magnitude of $i$ th trial. This index varies from minus infinity to +1 as the maximum improvement. Zero AE represents no adjustment in performance. It indicates how well the adjustments were made, on average, by each group (low- and highlearners) and during feedback and nonfeedback blocks. We divided the amount of improvement by the error in the previous trial based on the assumption that large adjustments are easier to accomplish than small ones. For example, the total adjustment from 800 to $400 \mathrm{msec}$ error is larger than 80 to $40 \mathrm{msec}$, but the latter is harder because it requires a more precise control of the time estimation. Considering that feedback was used to adjust performance, we expected the $\mathrm{AE}$ values to be higher on the feedback than on the nonfeedback blocks.

\section{EEG Recording and Analyses}

EEG signals were recorded by 64 active electrodes placed according to the extended 10-20 system of electrode placement and amplified by a BioSemi ActiveTwo amplifier (www.biosemi.com). The vertical and horizontal EOGs were recorded using four additional electrodes to monitor eye blinks and horizontal eye movements. The EEG signals were recorded with a sampling frequency of $512 \mathrm{~Hz}$, band-pass filtered between 0.16 and $100 \mathrm{~Hz}$. The task was presented on a PC using the MATLAB (The MathWorks, Inc., Natick, MA)-based toolbox Cogent 2000 (www.vislab.ucl.ac.uk/cogent.php). The EEG data were processed and analyzed using the following MATLABbased toolboxes: EEGLAB (Delorme \& Makeig, 2004) for data preprocessing and FieldTrip (Oostenveld, Fries, Maris, \& Schoffelen, 2011) for data analysis and statistical comparisons.

\section{Preprocessing}

The EEG data were re-referenced to a common average reference for ERP and wavelet analyses. The use of common average reference has been recommended for both ERPs and oscillations (Picton et al., 2000; Pfurtscheller \& Lopes da Silva, 1999) because it is a less biased method for comparing across scalp topographies. For the intersite synchronization analysis, we used the arithmetic average of the two earlobes because common average reference could introduce spurious correlations or interdependences between electrodes (Pereda, Quiroga, \& Bhattacharya, 2005). The data were high-pass filtered at $0.5 \mathrm{~Hz}$ and epoched from $-600 \mathrm{msec}$ before and $1000 \mathrm{msec}$ after feedback. The artifacts were treated in a semiautomated fashion: visual inspection was initially done to remove large muscle artifacts, followed by an independent component analysis for correcting the eye blink-related artifacts. Subsequent to the eye blink correction, epochs containing amplitudes exceeding $\pm 80 \mu \mathrm{V}$ were discarded for future analysis. The average number of artifact-free trials per participant (feedback trials only) was as follows: for the high-learners group, 103 (SD = 9.2) and 101 (6.8) trials for small and large errors, respectively, and for the low-learners group, 103 (1.3) and 100 (15.1) trials.

\section{ERP Analysis}

For the ERP analysis only, we applied a low-pass filter at $35 \mathrm{~Hz}$, and the filtered epoch of $200 \mathrm{msec}$ before feedback and 1 sec after feedback were averaged over trials to obtain the ERP signals. The ERPs were baseline ( -200 to 0 msec) subtracted.

\section{Time-Frequency Analysis}

The time-varying spectral content of the EEG data were estimated by Morlet wavelet based time-frequency representation (TFR). Each individual epoch, $600 \mathrm{msec}$ before feedback to $1 \mathrm{sec}$ after feedback, was convolved with complex Morlet wavelet on single-trial basis and subsequently averaged across trials. We used four cycle wavelets for lower frequencies $(4-35 \mathrm{~Hz})$ and seven cycle wavelets for higher frequencies $(>35 \mathrm{~Hz})$. To calculate the relative spectral power in each frequency, the TFR was normalized by dividing the spectral power in each frequency by its baseline value, from $-200 \mathrm{msec}$ to the onset of feedback.

\section{Intersite Synchronization Analysis}

Oscillatory activity represents local neuronal synchronization, whereas intersite or long-range neuronal synchronization can be measured by various connectivity-based indices (for a review, see Pereda et al., 2005). Here, we used a recently introduced measure of functional connectivity, the Weighted Phase Lag Index (WPLI), to study the patterns of phase synchronization from 3 to $67 \mathrm{~Hz}$ in steps of $4 \mathrm{~Hz}$. Vinck, Oostenveld, van Wingerden, Battaglia, and Pennartz (2011) introduced WPLI as an extension of the Phase Lag Index (PLI; Stam, Nolte, \& Daffertshofer, 2007) by weighting PLI according to the Imaginary Component of the Coherency (ImC; Nolte et al., 2004). The ImC is a measure of connectivity that is robust against volume conduction and uncorrelated sources (Nolte et al., 2004). However, Stam and colleagues (2007) remarked that ImC could be affected by the phase of the coherency: It is most effective for detecting synchronizations with a phase lag of a quarter cycle but is ineffective if the two signals are in phase or in phase opposition. The PLI index was proposed as an improvement over the 
ImC by measuring to what extent phase leads and lags between two channels are nonequiprobable (Stam et al., 2007). One problem with the PLI is that it is sensitive to noise because small perturbations can turn phase leads into lags and vice versa. To improve this index, the WPLI was developed by weighting the phase leads and lags by the magnitude of the imaginary component of the crossspectrum (Vinck et al., 2011). The WPLI was shown to be more robust to volume conduction and measurement noise than both ImC and PLI besides more sensitive to true interactions. In this study, we used a debiased version of WPLI after Vinck et al. (2011).

Each epoch $(-0.25$ to $1 \mathrm{sec})$ was divided into nonoverlapping five segments of $250 \mathrm{msec}$ each, and the WPLI was calculated on each of those segments. Considering that only a few studies analyzed the feedback-related connectivity (Cohen et al., 2011; Cavanagh et al., 2009, 2010; van de Vijver et al., 2011), we did not restrict our analysis to any specific electrode pair. First, we plotted the WPLI as a function of frequency, which led us to choose the beta band (19-23 Hz) as the frequency range of interest, because WPLI values peaked at these frequencies. For visualization, we used the "heads-in-head" plot (Nolte \& Muller, 2010; Nolte et al., 2004, 2008), which allows a simultaneous visualization of the connections between each electrode region. It also helped choosing the electrode pairs subsequently used for statistical comparisons.

\section{Statistical Analysis}

We used a nonparametric cluster permutation test, which is a biologically inspired statistical approach to analyze multidimensional EEG/MEG data in an exploratory framework but without compromising with the notorious issue of multiple comparisons (Maris \& Oostenveld, 2007). The method combines statistical significance with biological/ cognitive significance as follows. For an effect to be both statistically significant and biologically relevant, it needs to be found over a cluster of data points in all analyzed dimensions such as time, space (electrodes), and frequency, but an isolated significant difference found at a nonspecific data point would not be considered biologically relevant, therefore would not yield a significant cluster, even if it is highly significant statistically (i.e., $p<$ $.00001)$. This method is found to be as robust against Type I error as more conservative criteria like Bonferroni's correction, but with an improved sensitivity and without the requirement of hypothesis a priori. It has been successfully applied to EEG (Lindsen, Jones, Shimojo, \& Bhattacharya, 2010; Sandkuhler \& Bhattacharya, 2008) and MEG (Osipova et al., 2006).

The procedure (Maris \& Oostenveld, 2007) involves finding clusters and then calculating the cluster statistics in the following steps: (i) calculate a $t$ statistics (paired or independent) at each data point in the multidimensional space, (ii) apply threshold to these sample-specific statistics, (iii) construct connected clusters that exceed the threshold and have the same sign, (iv) calculate the cluster level statistics by summing the values of $t$ statistics over the cluster, (v) identify the maximum of the cluster level statistics, and (vi) evaluate this maximum value against a permutated distribution by calculating the exact probability that a cluster with the maximum cluster level statistic was observed under the assumption that the brain responses for the two compared conditions were not significantly different.

Here, we used this method to compare brain responses (ERPs and TFRs) to small and large error feedback for each group separately. The permutation distribution was derived from the statistic values of paired $t$ tests based on 500 random permutations. The probability threshold for the inclusion in the cluster was set at $p=.05$. We considered electrodes with a distance of less than $5 \mathrm{~cm}$ as neighbors, yielding on average 4.2 neighbors per electrode.

We used the cluster-based permutation to find the main differences between small and large error feedback and to define the ROIs on the multidimensional space (time, electrode, and frequency), which were subsequently analyzed by standard ANOVA as appropriate. Simple independent and paired $t$ tests were used to investigate simple effects. All statistical analyses were done using Statistical Package for the Social Sciences (Version 18.0; SPSS, Inc., Chicago, IL). The ANOVA was necessary to allow comparisons between groups and interactions, which were not accounted in the cluster permutation, because the latter was exclusively done to find the difference between small and large error feedback within each group.

The possible relationships between brain responses at the ROIs as defined by the cluster-based permutation test and performance during the nonfeedback blocks were investigated by Pearson's correlation. We were principally interested in the nonfeedback blocks because the performance on these trials was expected to represent the appropriateness and maintenance of the internal representation of the time interval learned during the feedback trials. It was not possible to evaluate the internal representation during the feedback blocks because on these trials the performance can be adjusted based on the feedback rather than on the internal representations of the learned time interval.

\section{RESULTS}

\section{Behavioral Results}

Figure 1 shows the performance as measured by the absolute error magnitude (Figure 1A), the response variability (Figure 1B), and the AE (Figure 1C) for both groups in successive blocks of trials. Across blocks, high-learners showed lower error magnitude, reduced variability, and higher AE than low-learners. The low-learners also showed higher performance decrements in the nonfeedback blocks, indicating a lower retention of the learned skill. 
These observations were confirmed as significant using a $2 \times 2 \times 2$ mixed MANOVA with Feedback (feedback vs. no feedback) and Stage (first vs. second halves of the task) as within-subjects factors, and Group (high-learners vs. low-learners) as the between-subject factor. There was a significant effect for Group, $F(2,29)=28.53, p<.001$, $\eta^{2}=0.66$, indicating that the performance was higher in the high- than in the low-learners group, and for Stage, $F(2,29)=19.82, p<.001, \eta^{2}=.58$, because the participants significantly improved their performance from the first to the second half of the task. Moreover, there was a significant effect of Feedback, $F(2,29)=28.14$, $p<.001, \eta^{2}=.66$, indicating that the performance in the feedback blocks was better than in the nonfeedback ones. We also found an interaction between Group and Feedback, $F(2,29)=4.28, p=.023, \eta^{2}=.23$, because the performance difference between feedback and nonfeedback was higher in the low-learners group. There was also an interaction between Feedback and Stage, $F(2$, 29) $=17.06, p<.001, \eta^{2}=.54$, because the performance difference between feedback and nonfeedback blocks was lower in the second half of the task as compared with the first.

A mixed ANOVA on the AE revealed that the highlearners presented a larger AE than the low-learners, $F(1$, $30)=64.90, p<.001, \eta^{2}=.68$, and that the AE increased from the first to the second half of the task, $F(1,30)=$ $10.31, p<.001, \eta^{2}=.26$. There was a significant effect of Feedback, $F(1,30)=32.14, p<.003, \eta^{2}=.52$, indicating that the $\mathrm{AE}$ was higher during the blocks with feedback. Furthermore, there was an interaction between Group and Feedback, $F(1,30)=4.21, p=.049, \eta^{2}=$ .12 , because the high-learners presented a lower difference in $\mathrm{AE}$ between feedback and nonfeedback blocks.

\section{ERP Analysis}

We compared the ERPs between two types of trial, with large error and small error feedback trials within each group separately by nonparametric cluster-based permutation statistic (see Methods). Both groups showed a frontal cluster between 200-300 msec with an FRNlike topography and a parietal cluster between 350 and $425 \mathrm{msec}$ (Figure 2A), the latter being larger in the lowlearners; following this parietal cluster (425-575 msec), a frontocentral cluster was observed in the high-learners only. Figure 2 shows the topographies of the cluster over time (a) and the waveforms averaged over the cluster electrodes (b).

The average ERP amplitudes over the electrodes and highlighted areas in Figure 2B were compared separately using a mixed $2 \times 2$ ANOVA with a within-subject factor, Error Magnitude (large vs. small error feedback), and a between-subject factor, Group (high-learners vs. lowlearners). Across groups, the FRN (averaged over Fz and FCz) amplitude was significantly higher (more negative) for the large error feedback compared with the small error feedback, $F(1,30)=36.91, p<.001, \eta^{2}=.552$. There was no effect of Group or interaction between Error Magnitude and Group $(F<1.5, p>.25)$. For the P300like component, we found a larger P300 following small than large error feedback, $F(1,30)=24.84, p<.001, \eta^{2}=$ .45 , but no effect for group or interaction $(F<1.5, p>$ $.25)$. Furthermore, the amplitude in left central electrodes (C3, C5) was higher for small than for large error feedback, $F(1,30)=19.97, p<.001, \eta^{2}=.40$, but no effects for Group or interactions $(F<1, p>.3)$. Finally, the ERP amplitude over left frontal electrode regions (FC3, F1, F3) was higher for small than large error feedback, $F(1$, $30)=13.294, p=.002$, but there were no significant differences between the two groups.

Next we correlated the ERP amplitudes of the ROIs as described earlier with the performance measures (mean error, variability, and AE) on the three nonfeedback blocks of the second half of the task (across participants). No significant correlation was observed.

\section{Time-Frequency Analysis}

We compared large and small error feedback for each group separately using nonparametric cluster permutation, and the results are shown in Figure 3A. For the high-learners, we found a significant beta band (17$24 \mathrm{~Hz}$, large $>$ small) cluster in the left sensorimotor region starting around $350 \mathrm{msec}$ after feedback. For the low-learners, we found an alpha band (10-13 Hz, large > small) cluster in the posterior parieto-occipital regions in the later stage of feedback processing, around $600 \mathrm{msec}$ after feedback. Figure 3B shows the time profiles of the beta band and alpha band effect for both groups for both large and small error feedback (Figure 3B).

A mixed ANOVA on the beta power over the cluster electrodes (highlighted in Figure 3A, left-hand side, high-learners cluster) revealed that the beta desynchronization was higher following small than large error feedback, $F(1,30)=50.27, p<.001, \eta^{2}=.63$, and that this difference was similar across groups $(F<1$, ns). Moreover, there was no significant effect for Time Window (early [375-525 $\mathrm{msec}]$ vs. late [600-800 $\mathrm{msec}]$ ) and Group $(F<2, n s)$. However, there was a significant interaction between Time Window and Group, $F(1,30)=$ $4.73, p=.038, \eta^{2}=.14$, which indicates that only the high-learners sustained the beta desynchronization in the late time window, 600-800 msec after feedback. Contrast $t$ tests indicated that the low-learners presented a significant increase in the beta power from the early to the late time window following small error feedback, $t(15)=-2.537, p=.023$, which was not observed in the high-learners group, $t(15)=.037, p=.971$, reinforcing that only the high-learners sustained this beta desynchronization. Following large error feedback, the beta power in those two time windows were not different $(t<1.5, n s)$. Further contrasts indicated that the two groups were only different in the late time window, because the high-learners 


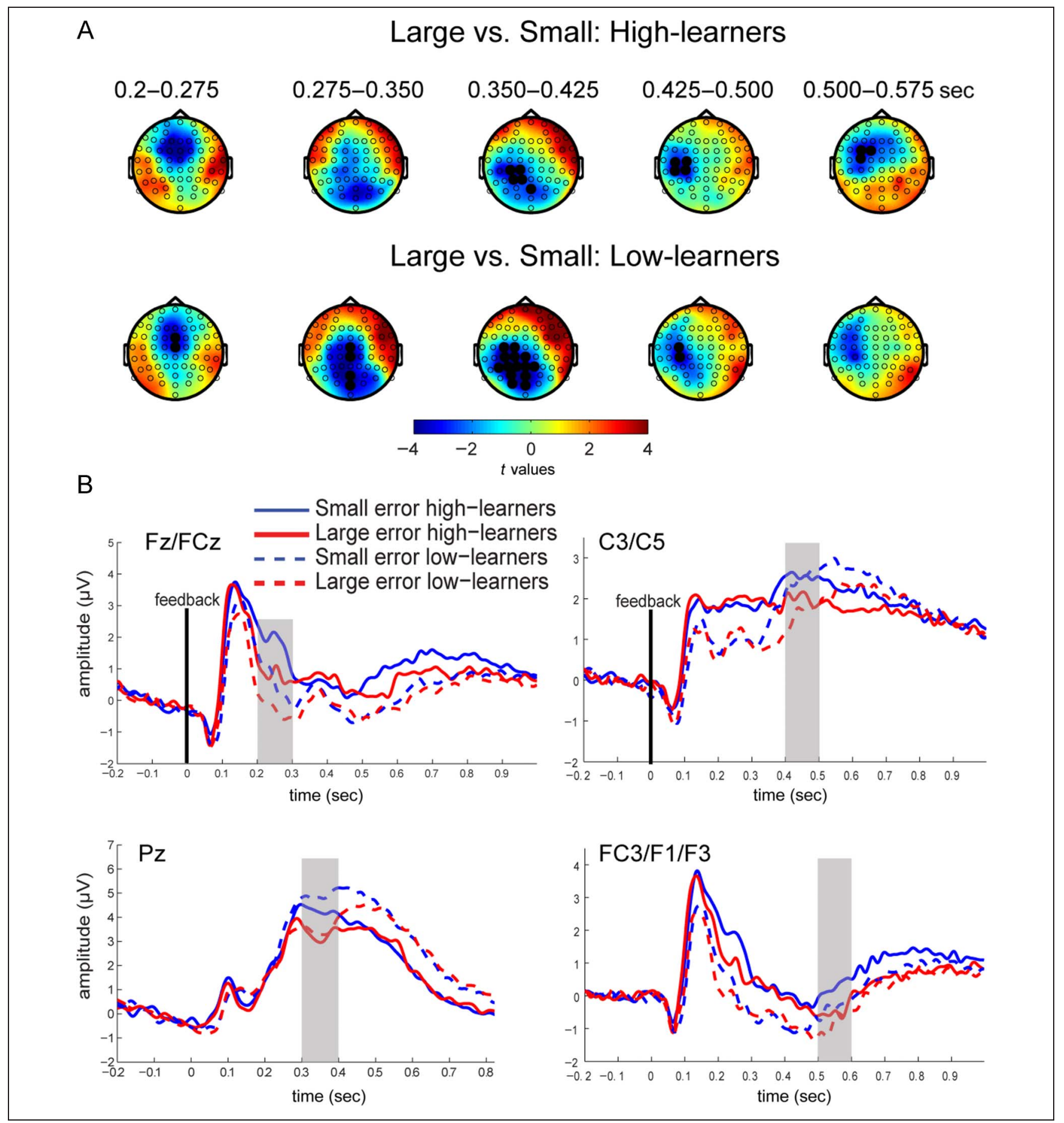

Figure 2. ERP cluster results. (A) Significant clusters of the nonparametric cluster randomization test comparing large and small errors for the two groups, high-learners (upper row) and low-learners (bottom row). The highlighted electrodes were significant during the whole time window displayed $(p<.05)$. (B) The ERP waveforms for each ROI averaging over the electrodes constituting the clusters. The time windows used for subsequent statistical analysis are highlighted in gray.

showed significantly lower beta power following small error feedback than the low-learners group, $t(30)=2.23, p=$ .033 , but not following large error feedback, $t(30)=1.83$, $p=.077$. The differences between groups in the early time window were nonsignificant $(t<1, n s)$.

Similar statistical analysis was conducted for alpha band power as well over the electrodes in the cluster (small vs. large error feedback) found in the low-learners group (Figure 3A, right-hand side). The alpha desynchronization was higher in small than large error feedback, $F(1,30)=25.91, p<.001, \eta^{2}=.46$, and the alpha power was lower in the early $(375-525 \mathrm{msec})$ than in the late (600-800 msec) time window, $F(1,30)=20.59, p<$ $.001, \eta^{2}=.40$. The high-learners showed significantly 


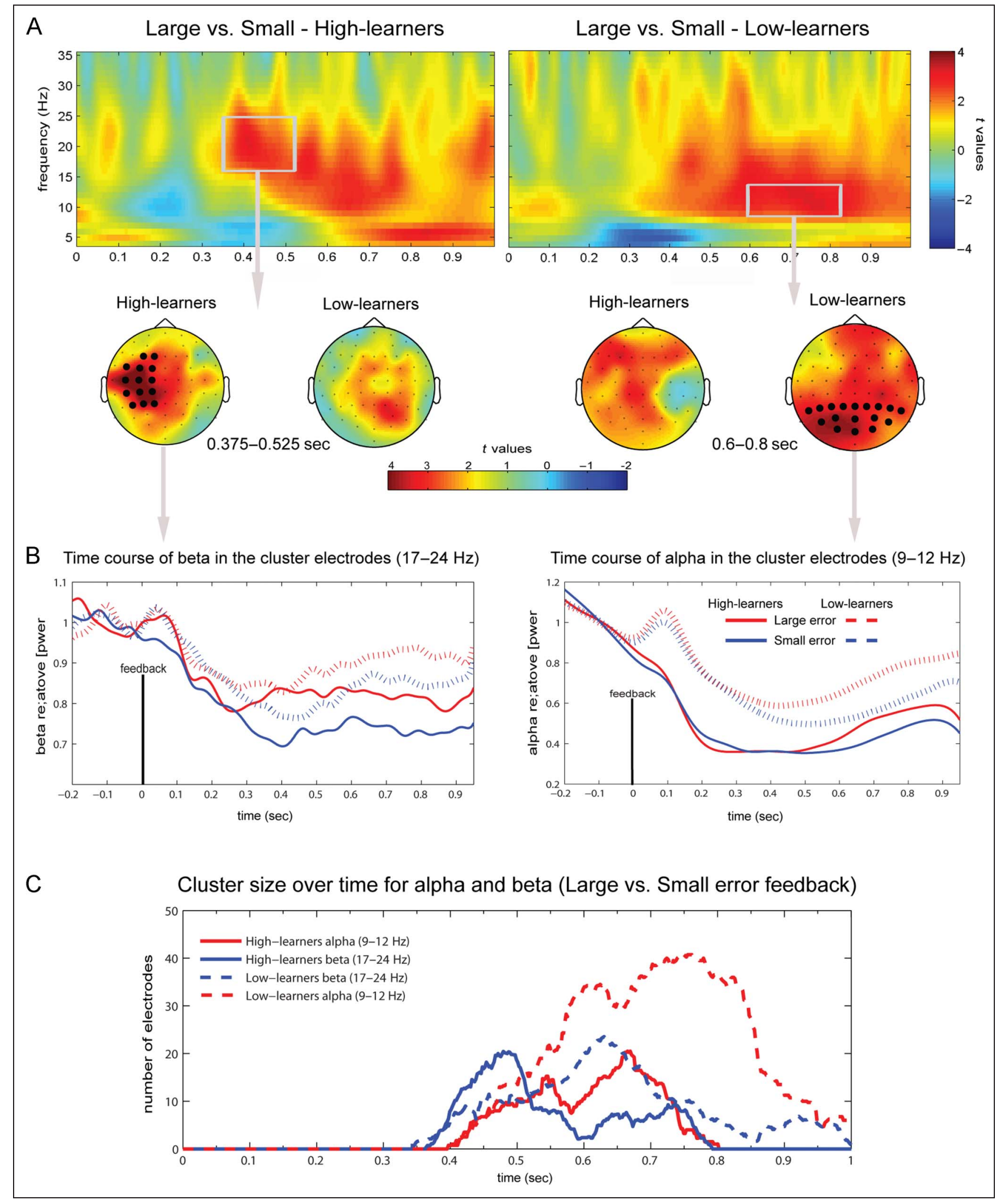

Figure 3. Oscillatory differences between small and large error feedback in high- and low-learners. (A) TFRs of the differences between large and small error feedback for high- and low-learners; the highlighted areas in the figures represent the strongest difference between small and large error feedback, whose topography is represented for high- and low-learners immediate below it. The larger dots in the topographical plots represent the electrodes that remained significant during the whole time window and frequency highlighted above. (B) Time course of beta (17-24 Hz) and alpha $(9-12 \mathrm{~Hz})$ averaged over the significant cluster electrodes (highlighted in the figure above them) for high- (solid lines) and low-learners (dashed lines) following large (red) and small (blue) error feedback. (C) Time-related changes in the size of the positive cluster, given by the average number of statistically significant electrodes $(p<.05)$ over the three main frequencies (upper alpha and beta) for high- (solid line) and low-learners (dashed line). 
larger desynchronization than the low-learners, $F(1,30)=$ $4.60, p=.040, \eta^{2}=.13$. Moreover, there was a significant interaction between Error Magnitude and Time Window, because there was a greater increase in the alpha power in the late time window for the large than for the small error feedback, which was associated with a sustained desynchronization.

Figure 3C shows the time-varying profiles of the size of these significant clusters. Whereas the low-learners group showed a high number of significant electrodes in the alpha band cluster that peaked between 600 and $800 \mathrm{msec}$ (dashed red line), the high-learners group presented a more localized, earlier cluster, around $400 \mathrm{msec}$ in the beta band. This result suggests that the differences between large and small error feedback processing are related to more a diffused representation in the lowlearners and to a more focused sensorimotor representation in the high-learners. For higher frequencies in the gamma range $(35-50 \mathrm{~Hz})$, we did not find any significant cluster for any comparisons (small vs. large, high-learners vs. low-learners).

\section{Connectivity/Synchronization Analysis}

Using the WPLI, we found an increase in phase synchronization from 0 to $250 \mathrm{msec}$ after feedback in relation to the baseline in the beta frequency range (17-24 Hz), only in the high-learners. The low-learners did not show any specific or clear synchronization pattern in any frequency band. In the high-learners, we observed a stronger intersite synchronization between the midfrontal (i.e., FCz) and the left central (C3) electrodes (Figure 4A, B). This is further demonstrated by the topographical distribution of the WPLI values between FCz and all other electrodes, and this effect was most conspicuous in the high-learners only. The averaged WPLI for the FCz-C3 pair in five nonoverlapping time segments spanning -250 to $1 \mathrm{sec}$ following feedback showed a large increase immediately after feedback for both small and large error feedback (Figure 4D). A mixed design 5 (Time Windows: -250 to 0, 0 to 250,250 to 500,500 to 750 , and 750 to $1000 \mathrm{msec}$ following feedback) $\times 2$ (Error Magnitude: small vs. large) ANOVA revealed a significant effect for Time, $F(2.37$, $30)=5.79, p=.003, \eta^{2}=.16$, but not for Error Magnitude, $F(1,30)=1.66, p=.207$. The high-learners group presented significantly larger WPLI values than the lowlearners group, $F(1,30)=5.75, p=.023, \eta^{2}=.16$. In addition, there was a significant interaction between Time and Group, $F(2.37,30)=3.94, p=.018, \eta^{2}=.12$, because the $\mathrm{FCz}-\mathrm{C} 3$ synchronization was increased in the high-learners group only (Figure 4D). Independent $t$ tests indicated that the high-learners presented significantly higher phase synchronization than the low-learners from 0 to 250 msec following both small, $t(30)=-3.148, p=$ .004 , and large error feedback, $t(30)=-3.342, p=.002$. There was no significant difference between groups for other time windows.
The WPLI between $\mathrm{FCz}-\mathrm{C} 3$ from 0 to 250 msec after feedback was found to be consistently correlated with most performance measures: error magnitude (small error feedback: $r=-0.701, p<.001$, see Figure 4C; large error feedback: $r=-0.674, p<.001$ ), error variability (small: $r=-.530, p=.002$; large: $r=-.569, p=$ .001 ), and AE (small: $r=.523, p=.002$; large: $r=.625, p<$ .001). These correlations suggest that the higher these two sites (FCz-C3) synchronized in the beta frequency range, the better was the performance (lower error, lower variability, and higher $\mathrm{AE}$ ).

\section{DISCUSSION}

Here we investigated the influence of the magnitude of the error feedback on electrophysiological responses and the further associations with learning performance. We used a simple task of time interval estimation, provided graded feedback (i.e., exact difference between the target and performance), and defined learning as a better maintenance of the time interval without a feedback. The electrophysiological differences between small and large errors were compared between high-learners and lowlearners, who were categorized based on their performance during trials with no feedback. The high-learners were not only better at performing the task, as expected because of our categorization criterion, but developed better internal representations of the learned time inter$\mathrm{val}$, as they showed lower differences in the performance during feedback and nonfeedback trials compared with the low-learners.

First, we discuss the differences between small and large errors in terms of ERPs, oscillations, and intersite synchronization. Second, we focus on how these different correlates were linked to learning the task and how they correlate with task performance.

\section{Small versus Large Error Feedback: ERPs, TFRs, and Phase Synchronization Differences}

\section{What Are the Differences in the Electrophysiological Correlates of Processing Small and Large Error Feedback?}

Similar to previous reports, we found that large errors were associated with a higher FRN and small errors with a higher P300 (Luft et al., 2013; van de Vijver et al., 2011; Sailer, Fischmeister, \& Bauer, 2010; Miltner et al., 1997). In addition, we observed that small errors generated a larger beta desynchronization, which is in contrast to studies with categorical feedback that showed higher beta synchronization following correct feedback (Luft et al., 2013; van de Vijver et al., 2011; Marco-Pallares et al., 2008).

Our ERP analysis showed that large error, as compared with small error, feedback was associated with a higher FRN. Studies investigating the link between FRN amplitude 
Figure 4. Phase

synchronization in response to feedback. (A) Head-in-head plot of the debiased WPLI in the beta range from 0 to 250 msec after feedback. Each channel is represented by a minitopographical plot showing the WPLI of it with all other channels. In the figure, $\mathrm{FCz}$ (the fourth midline electrode) has higher connections with left central electrodes, mainly C1 and C3, which are also synchronized with the CP3 and CP5. (B) The topographical distribution of the WPLI with FCz as the reference (" $R$ ") for high- and low-learners from 0 to 250 msec. (C) Correlation between average error on the last three nonfeedback blocks and the debiased WPLI for the $\mathrm{FCz}-\mathrm{C} 3$ pair from 0 to $250 \mathrm{msec}$ after feedback. (D) Mean and standard errors of the debiased WPLI in beta range for the analyzed segments after large and small error feedback for the highand low-learners groups.

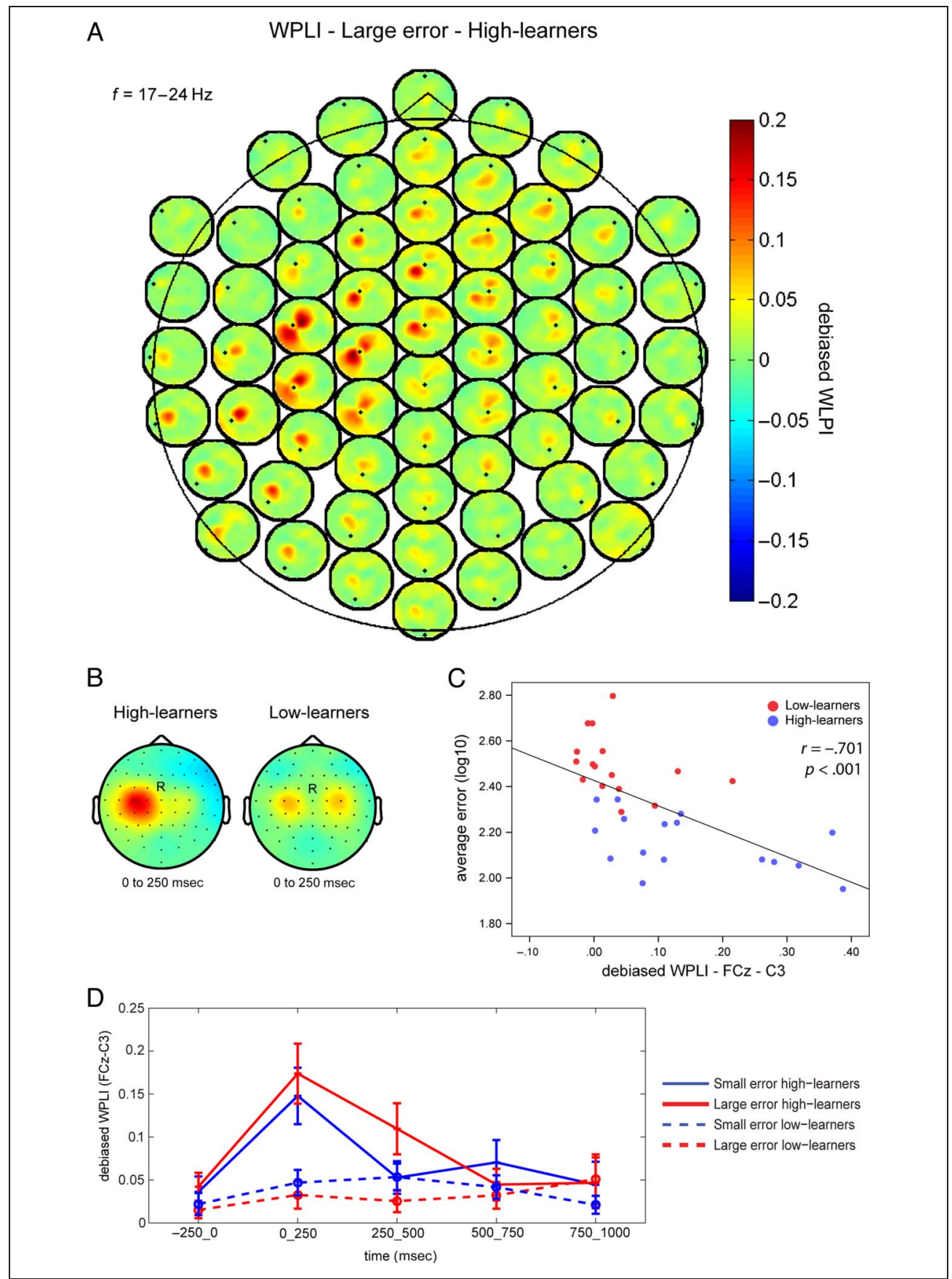

and reward/punishment magnitude have been inconclusive (Walsh \& Anderson, 2012), reporting no (Hajcak et al., 2006; Sato et al., 2005) or some effects (Kreussel et al., 2012; Donamayor, Marco-Pallares, Heldmann, Schoenfeld, \& Munte, 2011; Bellebaum et al., 2010; Wu \& Zhou, 2009). We also found a higher P300 for small as compared with large error feedback, which is in agreement with other studies showing higher P300 for positive compared with negative feedback (Luft et al., 2013; Sailer et al., 2010; van der Helden, Boksem, \& Blom, 2010; Wu \& Zhou, 2009; Bellebaum \& Daum, 2008; Hajcak et al., 2007).

In addition, previous studies showed that the P300 is sensitive to the reward magnitude (Wu \& Zhou, 2009;
Sato et al., 2005), suggesting that, although the FRN reflects a mechanism for evaluating whether the feedback is consistent or not with the expectations, the P300 represents a later process of outcome evaluation that is topdown regulated for the allocation of attentional resources. In our study, we originally provided graded feedback, which was later divided into two categories, large error or small error, according to each individual's performance. Therefore, it might also be possible that, rather than showing a sensitivity to error magnitude, the participants engaged in a process of an implicit categorization (Seger, 2008) in which different errors were categorized as good or bad. 
We also found a left-lateralized ERP difference between small and large error feedback over sensorimotor electrode regions. A similar pattern is observed when comparing losses versus wins in a competitive game (Cohen \& Ranganath, 2007), suggesting that feedback information may be processed in the motor cortex, whose role is to strengthen the winning responses and weaken the losses. In both studies (Cohen \& Ranganath, 2007; and the present), spatial resolution is limited to determine accurately whether the hand area was involved, but the electrodes (C3 and C5) are commonly used to investigate motor responses (Neuper, Scherer, Wriessnegger, \& Pfurtscheller, 2009; Neuper, Scherer, Reiner, \& Pfurtscheller, 2005). We suggest that our effect represents a similar process of strengthening/weakening of the neural representation associated with good/poor performance. Another possibility is that we learn not by a single prediction error for the whole action but by subsets of prediction error for each subcomponent of the task. An fMRI study (Gershman, Pesaran, \& Daw, 2009) using a bimanual probabilistic task in which the right- and left-hand responses were independently rewarded found that the system decomposes the prediction errors in small subaction spaces. They found that the striatal responses to the prediction error were contralateral to the response hand, according to the effector space (right or left hand).

In brief, we found three main ERP effects related with the magnitude (small/large) of the error feedback: (1) higher FRN following large error feedback reflecting error detection, (2) higher P300 following small error feedback reflecting attention and memory allocation, and (3) higher sensorimotor positivity following small error feedback reflecting motor reinforcement.

The results so far support that, although large errors evoked larger FRN, small error feedback seems to allocate more resources related to attention and memory processes. This hypothesis was further supported by our analysis based on neural oscillations. Small error feedback was associated with a higher decrease in spectral power in the alpha and beta frequency bands, indicating larger brain activation in response to small error feedback. This interpretation is based on previous studies showing that alpha and beta desynchronizations are inversely related with sensorimotor activation (Neuper, Wortz, \& Pfurtscheller, 2006; Pfurtscheller \& Lopes da Silva, 1999; Pfurtscheller \& Neuper, 1997). Previous experiments found an increase in the beta power in response to positive feedback and rewards (HajiHosseini et al., 2012; MarcoPallares et al., 2008; Cohen et al., 2007; Hallschmid, Mölle, Fischer, \& Born, 2002) and others that found a desynchronization in beta range following incorrect performance feedback (Luft et al., 2013; van de Vijver et al., 2011). The former studies have found synchronization in the beta or low-gamma range $(20-40 \mathrm{~Hz})$ in response to rewards. On the basis of previous animal experiments demonstrating increased beta, gamma, and high-gamma oscillations in striatum in response to actions associated with rewards
(Berke, 2009; Courtemanche, Fujii, \& Graybiel, 2003), it was suggested that beta and gamma oscillations following positive feedback reflect the activation of reward-related areas in the brain.

There are three main factors that may indicate that the beta power increase found in these studies are unlikely to represent the same phenomenon as the one we found in the present article and that Luft et al. (2013) and van de Vijver et al. (2011) also found. First, the paradigm adopted in the Luft et al. (2013) and van de Vijver et al. (2011) studies is a time estimation task whose feedback represents the accuracy of the response (correct or incorrect) rather than a reward. Second, the topography of the beta oscillations differs, because the reward studies found a frontal or midfrontal topography whereas the latter studies have a sensorimotor left-lateralized topography, which is the same topography found in this study. Third, the time estimation studies looked at a slightly lower frequency range $(17-24 \mathrm{~Hz})$ than the reward studies $(20-40 \mathrm{~Hz})$. We suggest that, for performance feedback, this desynchronization may reflect a sensorimotor reorganization in response to error. Importantly, Luft et al. (2013) found that the higher the desynchronization, the better the participants learned or consolidated their time estimations when feedback was not available.

Considering that motor preparation and imagery are associated with the beta desynchronization over the contralateral motor areas (Pfurtscheller, Neuper, Ramoser, \& Muller-Gerking, 1999; Pfurtscheller \& Neuper, 1997) and that tiny adjustments are harder to accomplish than large ones, this sensorimotor operation may reflect motor imagery processes used to retrieve and restructure the last trial. In our present study, we support the idea that the beta desynchronization over the left-sensorimotor areas may reflect a process of sensorimotor reorganization based on feedback. Higher beta desynchronization in response to small rather than large errors might be related to how much effort a small and a large error requires for correction. It is common sense that reaching perfection requires more than the initial learning. Therefore, a small error, which requires a precise correction, has the potential to trigger more sophisticated error correction mechanisms than a large error where the scope for correction is larger.

\section{High- versus Low-learners: ERPs, Oscillations, and Phase Synchronization Differences and Correlation with Performance}

\section{Is There a Difference between High-and Low-learners in Relation to the Way They Process Graded Feedback?}

Our high-learners were not only better performers (reduced error and variability), but also better at retaining an internal representation of the learned skill. In addition, the highlearners were better with performance adjustment. The two learning groups differed mainly in terms of neuronal 
oscillations and intersite synchronization, but not in relation to ERPs.

In our results on oscillations, the topographies of the clusters found for the small versus large error feedback comparisons indicate low-learners presented a larger overall desynchronization following small error feedback, mainly in the alpha band, whereas the high-learners group showed an earlier and more localized cluster that starts in the left centrofrontal areas around $400 \mathrm{msec}$ in the beta band and finally spreads to midparietal and temporal areas in the alpha frequency band. This result might indicate that low-learners presented a more general attention effect related to feedback, whereas the high-learners showed a more specific pattern that could be related to the brain processes used to learn the task. Earlier we have suggested that the beta desynchronization over motor areas plays an important role in learning from error feedback. This account is further supported by our result showing larger beta desynchronization in the high-learners, and this effect was most conspicuous for small error feedback. Furthermore, studies using transcranial direct current stimulation (Galea, Vazquez, Pasricha, de Xivry, \& Celnik, 2011; Reis et al., 2009) indicated that stimulating the primary motor area promoted a better retention of a motor skill, which means a better learning. Therefore, these results altogether support the notion that higher activations over the motor cortex are associated with better learning. In addition, we suggest that the involvement of these areas for feedback processing is relevant for consolidating learned skills.

We found that only high-learners showed increased connectivity between midfrontal (FCz) and left central (C3) sites following both small and large error feedback. This midfrontal region has been widely implicated in performance monitoring (Balconi \& Scioli, 2011; Cohen et al., 2011; Cavanagh et al., 2009, 2010; Luu et al., 2003; Holroyd \& Coles, 2002) for correcting actions (Cohen \& Ranganath, 2007; Ullsperger \& von Cramon, 2006). Cavanagh et al. (2009) showed that the connectivity between FCz and the lateral pFC (F5/6) was increased after error trials and was further predictive of post-error adjustment. Moreover, a new framework for understanding the neural mechanisms of feedback guided learning, proposed by Cohen et al. (2011), suggested that learning could be associated with increased synchronization between stimulus-specific and motor areas involved in the learned skill. In our study, we found that midfrontal region (FCz) was synchronized with the left central area (C3) after the presentation of both small and large error feedback. This connectivity pattern was not present in the lowlearners group, suggesting that learning might strengthen the synchronization between these two brain regions allowing a more efficient error adjustment or that the synchronization between those two areas in response to feedback facilitates learning. Our current study could not dissociate between these two possibilities, and future studies are needed to establish whether learning is an effect or cause of synchronization between these areas. Interestingly, this synchronization, between FCz and C3 in the beta band, was highly correlated with performance in the nonfeedback blocks, indicating that the more these areas communicate after feedback, the better is the consolidation of the learned skill.

Taken together, these results suggest that high- and lowlearners differed in the way they processed small and large error feedback on electrodes and time windows defined based on the differences between small and large error feedback. Therefore, differences in other areas and other frequency bands that were similar for small and large error feedback may have been overlooked. Further studies comparing the brain responses to feedback between good and poor learners may be necessary to find differences between those two groups that were not linked to processing of distinct performance feedback magnitude.

\section{Conclusion}

In this study, we showed the effect of graded feedback on brain responses and how it differed between individuals with distinct learning abilities in a time estimation task. We conclude that small rather than large error feedback is associated with higher alpha and beta desynchronization, possibly associated with a more sophisticated process for correcting errors. Individual differences in learning from graded feedback are mainly evident in the oscillatory correlates of processing of small rather than of large errors. Finally, we found that phase synchronization in the beta frequency range between midfrontal and left sensorimotor-related electrodes was correlated with how well the participants were able to perform when no feedback was available.

\section{Acknowledgments}

We are thankful to Job Lindsen for the advice on data analysis and to Dr. Stuart Derbyshire for proof reading this manuscript. C. D. B. L. was supported by CAPES Foundation (PDEE programme, Process number 1428-10-1) to carry out the experiment at Goldsmiths, University of London. J. B. was partially supported by the grant, TEC-2012-38453-C04-03 of the Spanish Ministry of Economic and Competitiveness.

Reprint requests should be sent to Caroline Di Bernardi Luft, Department of Psychology, Goldsmiths, University of London, New Cross-SE14 6NW, London, UK, or via e-mail: caroluft21@ yahoo.com.br.

\section{REFERENCES}

Balconi, M., \& Scioli, S. (2011). Error monitoring functions in response to an external feedback when an explicit judgement is required ERP modulation and cortical source localisation. International Journal of Psychophysiology, 83, 295-301.

Bellebaum, C., \& Daum, I. (2008). Learning-related changes in reward expectancy are reflected in the feedback-related negativity. European Journal of Neuroscience, 27, 1823-1835. 
Bellebaum, C., Polezzi, D., \& Daum, I. (2010). It is less than you expected: The feedback-related negativity reflects violations of reward magnitude expectations. Neuropsychologia, 48, 3343-3350.

Berke, J. D. (2009). Fast oscillations in cortical-striatal networks switch frequency following rewarding events and stimulant drugs. European Journal of Neuroscience, 30, 848-859.

Burkhard, D. G., Patterson, J., \& Rapue, R. (1967). Effect of film feedback on learning the motor skills of karate. Perceptual $\varepsilon$ Motor Skills, 25, 65-69.

Carter, C. S., Braver, T. S., Barch, D. M., Botvinick, M. M., Noll, D., \& Cohen, J. D. (1998). Anterior cingulate cortex, error detection, and the online monitoring of performance. Science, 280, 747-749.

Cavanagh, J. F., Cohen, M. X., \& Allen, J. J. (2009). Prelude to and resolution of an error: EEG phase synchrony reveals cognitive control dynamics during action monitoring. Journal of Neuroscience, 29, 98-105.

Cavanagh, J. F., Frank, M. J., Klein, T. J., \& Allen, J. J. (2010). Frontal theta links prediction errors to behavioral adaptation in reinforcement learning. Neuroimage, 49, 3198-3209.

Cavanagh, J. F., Zambrano-Vazquez, L., \& Allen, J. J. (2012). Theta lingua franca: A common mid-frontal substrate for action monitoring processes. Psychophysiology, 49, 220-238.

Cohen, M. X., Elger, C. E., \& Ranganath, C. (2007). Reward expectation modulates feedback-related negativity and EEG spectra. Neuroimage, 35, 968-978.

Cohen, M. X., \& Ranganath, C. (2007). Reinforcement learning signals predict future decisions. Journal of Neuroscience, 27, 371-378.

Cohen, M. X., Wilmes, K., \& Vijver, I. (2011). Cortical electrophysiological network dynamics of feedback learning. Trends in Cognitive Sciences, 15, 558-566.

Courtemanche, R., Fujii, N., \& Graybiel, A. M. (2003). Synchronous, focally modulated beta-band oscillations characterize local field potential activity in the striatum of awake behaving monkeys. Journal of Neuroscience, 23, 11741-11752.

Delorme, A., \& Makeig, S. (2004). EEGLAB: An open source toolbox for analysis of single-trial EEG dynamics including independent component analysis. Journal of Neuroscience Methods, 134, 9-21.

Diedrichsen, J., White, O., Newman, D., \& Lally, N. (2010). Use-dependent and error-based learning of motor behaviors. Journal of Neuroscience, 30, 5159-5166.

Donamayor, N., Marco-Pallares, J., Heldmann, M., Schoenfeld, M. A., \& Munte, T. F. (2011). Temporal dynamics of reward processing revealed by magnetoencephalography. Human Brain Mapping, 32, 2228-2240.

Galea, J. M., Vazquez, A., Pasricha, N., de Xivry, J. J., \& Celnik, P. (2011). Dissociating the roles of the cerebellum and motor cortex during adaptive learning: The motor cortex retains what the cerebellum learns. Cerebral Cortex, 21, 1761-1770.

Gehring, W. J., \& Willoughby, A. R. (2002). The medial frontal cortex and the rapid processing of monetary gains and losses. Science, 295, 2279-2282.

Gershman, S. J., Pesaran, B., \& Daw, N. D. (2009). Human reinforcement learning subdivides structured action spaces by learning effector-specific values. Journal of Neuroscience, 29, 13524-13531.

Hajcak, G., Moser, J. S., Holroyd, C. B., \& Simons, R. F. (2006). The feedback-related negativity reflects the binary evaluation of good versus bad outcomes. Biological Psychology, 71, 148-154.

Hajcak, G., Moser, J. S., Holroyd, C. B., \& Simons, R. F. (2007). It's worse than you thought: The feedback negativity and violations of reward prediction in gambling tasks.

Psychophysiology, 44, 905-912.
HajiHosseini, A., Rodriguez-Fornells, A., \& Marco-Pallares, J. (2012). The role of beta-gamma oscillations in unexpected rewards processing. Neuroimage, 60, 1678-1685.

Hallschmid, M., Mölle, M., Fischer, S., \& Born, J. (2002). EEG synchronization upon reward in man. Clinical Neurophysiology: Official Journal of the International Federation of Clinical Neurophysiology, 113, 1059-1065.

Holroyd, C. B., \& Coles, M. G. (2002). The neural basis of human error processing: Reinforcement learning, dopamine, and the error-related negativity. Psychological Review, 109, 679-709.

Holroyd, C. B., \& Krigolson, O. E. (2007). Reward prediction error signals associated with a modified time estimation task. Psychophysiology, 44, 913-917.

Kawashima, R., Tajima, N., Yoshida, H., Okita, K., Sasaki, T., Schormann, T., et al. (2000). The effect of verbal feedback on motor learning-A PET study. Positron emission tomography. Neuroimage, 12, 698-706.

Kreussel, L., Hewig, J., Kretschmer, N., Hecht, H., Coles, M. G., \& Miltner, W. H. (2012). The influence of the magnitude, probability, and valence of potential wins and losses on the amplitude of the feedback negativity. Psychophysiology, 49, 207-219.

Kreussel, L., Hewig, J., Kretschmer, N., Hecht, H., Coles, M. G., \& Miltner, W. H. (2013). How bad was it? Differences in the time course of sensitivity to the magnitude of loss in problem gamblers and controls. Behavioural Brain Research, 247, 140-145.

Ladouceur, C. D., Dahl, R. E., \& Carter, C. S. (2007). Development of action monitoring through adolescence into adulthood: ERP and source localization. Developmental Science, 10, 874-891.

Lewis, P. A., \& Miall, R. C. (2003). Brain activation patterns during measurement of sub- and supra-second intervals. Neuropsychologia, 41, 1583-1592.

Lindsen, J. P., Jones, R., Shimojo, S., \& Bhattacharya, J. (2010). Neural components underlying subjective preferential decision making. Neuroimage, 50, 1626-1632.

Luft, C. D. B., Nolte, G., \& Bhattacharya, J. (2013). High-learners present larger mid-frontal theta power and connectivity in response to incorrect performance feedback. Journal of Neuroscience, 33, 2029-2038.

Luu, P., Tucker, D. M., Derryberry, D., Reed, M., \& Poulsen, C. (2003). Electrophysiological responses to errors and feedback in the process of action regulation. Psychological Science, 14, 47-53.

Marco-Pallares, J., Cucurell, D., Cunillera, T., Garcia, R., AndresPueyo, A., Munte, T. F., et al. (2008). Human oscillatory activity associated to reward processing in a gambling task. Neuropsychologia, 46, 241-248.

Maris, E., \& Oostenveld, R. (2007). Nonparametric statistical testing of EEG- and MEG-data. Journal of Neuroscience Methods, 164, 177-190.

Mars, R. B., Bruijn, E. R. A., Hulstijn, W., Miltner, W. H. R., \& Coles, M. G. H. (2004). What if I told you: "You were wrong"?: Brain potentials and behavioral adjustments elicited by feedback in a time-estimation task. In U. M. Falkenstein (Ed.), Errors, conflicts, and the brain: Current opinions on performance monitoring (pp. 129-134). Leipzig: MPI of Cognitive Neuroscience.

Masters, R. S., Maxwell, J. P., \& Eves, F. F. (2009). Marginally perceptible outcome feedback, motor learning and implicit processes. Consciousness and Cognition, 18, 639-645.

Miltner, W. H. R., Braun, C., \& Coles, M. G. H. (1997). Eventrelated brain potentials following incorrect feedback in a time-estimation task: Evidence for a generic neural system for error detection. Journal of Cognitive Neuroscience, 9, 788-798. 
Neuper, C., Scherer, R., Reiner, M., \& Pfurtscheller, G. (2005) Imagery of motor actions: Differential effects of kinesthetic and visual-motor mode of imagery in single-trial EEG. Brain Research, Cognitive Brain Research, 25, 668-677.

Neuper, C., Scherer, R., Wriessnegger, S., \& Pfurtscheller, G. (2009). Motor imagery and action observation: Modulation of sensorimotor brain rhythms during mental control of a brain-computer interface. Clinical Neurophysiology, 120, 239-247.

Neuper, C., Wortz, M., \& Pfurtscheller, G. (2006). ERD/ERS patterns reflecting sensorimotor activation and deactivation. Progress in Brain Research, 159, 211-222.

Nolte, G., Bai, O., Wheaton, L., Mari, Z., Vorbach, S., \& Hallett, M. (2004). Identifying true brain interaction from EEG data using the imaginary part of coherency. Clinical Neurophysiology, 115, 2292-2307.

Nolte, G., \& Muller, K. R. (2010). Localizing and estimating causal relations of interacting brain rhythms. Frontiers in Human Neuroscience, 4, 209.

Nolte, G., Ziehe, A., Nikulin, V. V., Schlogl, A., Kramer, N., Brismar, T., et al. (2008). Robustly estimating the flow direction of information in complex physical systems. Physical Review Letters, 100, 234101.

Oostenveld, R., Fries, P., Maris, E., \& Schoffelen, J. M. (2011). FieldTrip: Open source software for advanced analysis of MEG EEG, and invasive electrophysiological data. Computational Intelligence and Neuroscience, 2011, 156869.

Osipova, D., Takashima, A., Oostenveld, R., Fernandez, G., Maris, E., \& Jensen, O. (2006). Theta and gamma oscillations predict encoding and retrieval of declarative memory. Journal of Neuroscience, 26, 7523-7531.

Pereda, E., Quiroga, R. Q., \& Bhattacharya, J. (2005). Nonlinear multivariate analysis of neurophysiological signals. Progress in Neurobiology, 77, 1-37.

Pfurtscheller, G., \& Lopes da Silva, F. H. (1999). Event-related EEG/MEG synchronization and desynchronization: Basic principles. Clinical Neurophysiology, 110, 1842-1857.

Pfurtscheller, G., \& Neuper, C. (1997). Motor imagery activates primary sensorimotor area in humans. Neuroscience Letters, 239, 65-68.

Pfurtscheller, G., Neuper, C., Ramoser, H., \& Muller-Gerking, J. (1999). Visually guided motor imagery activates sensorimotor areas in humans. Neuroscience Letters, 269, 153-156.

Picton, T. W., Bentin, S., Berg, P., Donchin, E., Hillyard, S. A., Johnson, R., Jr., et al. (2000). Guidelines for using human event-related potentials to study cognition: Recording standards and publication criteria. Psychophysiology, 37, 127-152.

Potts, G. F., Martin, L. E., Kamp, S. M., \& Donchin, E. (2011). Neural response to action and reward prediction errors: Comparing the error-related negativity to behavioral errors and the feedback-related negativity to reward prediction violations. Psychophysiology, 48, 218-228.

Reis, J., Schambra, H. M., Cohen, L. G., Buch, E. R., Fritsch, B., Zarahn, E., et al. (2009). Noninvasive cortical stimulation enhances motor skill acquisition over multiple days through an effect on consolidation. Proceedings of the National Academy of Sciences, U.S.A., 106, 1590-1595.

Sailer, U., Fischmeister, F. P., \& Bauer, H. (2010). Effects of learning on feedback-related brain potentials in a decisionmaking task. Brain Research, 1342, 85-93.
Sandkuhler, S., \& Bhattacharya, J. (2008). Deconstructing insight: EEG correlates of insightful problem solving. PLoS One, 3, e1459.

Santesso, D. L., Bogdan, R., Birk, J. L., Goetz, E. L., Holmes, A. J., \& Pizzagalli, D. A. (2012). Neural responses to negative feedback are related to negative emotionality in healthy adults. Social Cognitive and Affective Neuroscience, 7, 794-803.

Sato, A., Yasuda, A., Ohira, H., Miyawaki, K., Nishikawa, M., Kumano, H., et al. (2005). Effects of value and reward magnitude on feedback negativity and P300. NeuroReport, 16, 407-411

Seger, C. A. (2008). How do the basal ganglia contribute to categorization? Their roles in generalization, response selection, and learning via feedback. Neuroscience and Biobehavioral Reviews, 32, 265-278.

Stam, C. J., Nolte, G., \& Daffertshofer, A. (2007). Phase lag index: Assessment of functional connectivity from multi channel EEG and MEG with diminished bias from common sources. Human Brain Mapping, 28, 1178-1193.

Tucker, D. M., Luu, P., Frishkoff, G., Quiring, J., \& Poulsen, C. (2003). Frontolimbic response to negative feedback in clinical depression. Journal of Abnormal Psychology, 112, 667-678.

Ullsperger, M., \& von Cramon, D. Y. (2006). How does error correction differ from error signaling? An event-related potential study. Brain Research, 1105, 102-109.

van de Vijver, I., Ridderinkhof, K. R., \& Cohen, M. X. (2011). Frontal oscillatory dynamics predict feedback learning and action adjustment. Journal of Cognitive Neuroscience, 23, 4106-4121.

van der Helden, J., Boksem, M. A., \& Blom, J. H. (2010). The importance of failure: Feedback-related negativity predicts motor learning efficiency. Cerebral Cortex, 20, 1596-1603.

Van Veen, V., \& Carter, C. S. (2002). The timing of actionmonitoring processes in the anterior cingulate cortex. Journal of Cognitive Neuroscience, 14, 593-602.

Vinck, M., Oostenveld, R., van Wingerden, M., Battaglia, F., \& Pennartz, C. M. (2011). An improved index of phasesynchronization for electrophysiological data in the presence of volume-conduction, noise and sample-size bias. Neuroimage, 55, 1548-1565.

Walsh, M. M., \& Anderson, J. R. (2012). Learning from experience: Event-related potential correlates of reward processing, neural adaptation, and behavioral choice. Neuroscience and Biobehavioral Reviews, 36, 1870-1884.

Wolpert, D. M., Diedrichsen, J., \& Flanagan, J. R. (2011). Principles of sensorimotor learning. Nature Reviews Neuroscience, 12, 739-751.

Wu, Y., \& Zhou, X. (2009). The P300 and reward valence, magnitude, and expectancy in outcome evaluation. Brain Research, 1286, 114-122.

Wulf, G., Chiviacowsky, S., Schiller, E., \& Avila, L. T. (2010). Frequent external-focus feedback enhances motor learning. Frontiers in Psychology, 1, 190.

Yeung, N., \& Sanfey, A. G. (2004). Independent coding of reward magnitude and valence in the human brain. Journal of Neuroscience, 24, 6258-6264.

Zhou, Z., Yu, R., \& Zhou, X. (2010). To do or not to do? Action enlarges the FRN and P300 effects in outcome evaluation. Neuropsychologia, 48, 3606-3613. 